\title{
The Intersectional Experiences of Black Women in Computing
}

\author{
Yolanda A. Rankin ** \\ Florida State University \\ Tallahassee, Florida, USA \\ yrankin@fsu.edu
}

\author{
Jakita O. Thomas \\ Auburn University \\ Auburn, Alabama, USA \\ jnt0020@auburn.edu
}

\begin{abstract}
Efforts to broaden participation in computing have led to genderfocused interventions intended to increase the number of women in the field of computing. However, such efforts have failed to significantly increase the percentage of Black women in computing. For example, only $1 \%$ of the 28,884 bachelor's degrees in computing were awarded to Black women in 2018. Moreover, too few empirical studies have intentionally explored the lived experiences of Black women, an often overlooked and understudied population in the computing ecosystem. In this paper, we introduce intersectionality - the complex overlap of socially constructed identities such as race, gender, class, sexuality, etc. - as a theoretical framework and springboard for exploring the lived experiences of Black women in computing. We interview 14 Black women in various stages of the computing ecosystem (undergraduate students, graduate students and early career professionals) to understand how intersectionality influences their ability to persist in computing. Preliminary findings from the analysis of the 14 interviews provides insights into how the interlocking systems of oppression (i.e., gendered racism) play out in computing education and negatively impact the recruitment and retention of Black women in the field of computing.
\end{abstract}

\section{CCS CONCEPTS}

- Social and professional topics $\rightarrow$ Professional topics; Computing education; Adult education.

\section{KEYWORDS}

Black women, computing, gendered racism, intersectionality

\section{ACM Reference Format:}

Yolanda A. Rankin and Jakita O. Thomas. 2020. The Intersectional Experiences of Black Women in Computing. In The 51st ACM Technical Symposium on Computer Science Education (SIGCSE '20), March 11-14, 2020, Portland, OR, USA. ACM, New York, NY, USA, 7 pages. https://doi.org/10.1145/3328778. 3366873

${ }^{*}$ Both authors contributed equally to this research.

Permission to make digital or hard copies of all or part of this work for personal or classroom use is granted without fee provided that copies are not made or distributed for profit or commercial advantage and that copies bear this notice and the full citation on the first page. Copyrights for components of this work owned by others than ACM must be honored. Abstracting with credit is permitted. To copy otherwise, or republish, to post on servers or to redistribute to lists, requires prior specific permission and/or a fee. Request permissions from permissions@acm.org.

SIGCSE '20, March 11-14, 2020, Portland, OR, USA

(C) 2020 Association for Computing Machinery.

ACM ISBN 978-1-4503-6793-6/20/03 . \$ \$15.00

https://doi.org/10.1145/3328778.3366873

\section{INTRODUCTION}

Though the number of women receiving bachelor's degrees in computing (i.e., Computer Science, Information Technology, Computer Engineering, Data Science, etc.) has increased, a closer look reveals that the percentage of U.S. Black women in computing has significantly dropped over a ten-year period (2004 -2014), highlighting the underrepresentation of U.S. Black women in the field of computing[1].The literature reveals that a number of $\mathrm{K}$ 18 interventions (e.g., culturally relevant computing, gaming, efashion, recipes, etc.) have been designed to increase the representation of Black women and girls in computing in the U.S. [13][12] [29][28][37][31][34] [30] [33]. Despite these best efforts, the needle seems to have barely moved in increasing the representation of Black women in the field of computing [1] [40].

Instead, the primary goals have been to recruit and retain women in the computing ecosystem using gender-focused efforts intended to increase the number of women who are also members of ethnically underrepresented populations [2], [6][14][17][32]. This approach makes one flawed assumption - that by tackling the issue of gender which correlates to a larger segment of the U.S. population, the issue of race becomes null and void [38]. This is indicative of gendered racism, a specific type of oppression that Black women and other women of color experience due to the intersection of race and gender[8]. Because women of color share the same gender as white women but differ in race, they are subjugated to a different reality and set of social injustices that are often ignored by gender-focused efforts [7][8] [10][3][20]. Subsequently, these gender-focused efforts have fallen short of increasing the number of Black women in computing because of their failure to acknowledge or appreciate how intersectionality (the complex overlapping social constructs of gender, race, ethnicity, class, etc.) has shaped the lived experiences of women of color navigating the computing ecosystem [5][4][8][9] [15][18][19][22][25][26][27][38][39]. Ignoring the intersectional experiences of women of color leads to incorrect generalizations, which then leads to ineffective approaches for increasing the representation and retention of Black women in computing.

In response to this dilemma, we utilize intersectionality as a theoretical framework to explore the overlapping social constructs of race and gender that shape the everyday experiences of 14 Black women navigating the computing ecosystem. Our goals are to: 1) critique the dominant narrative in Computer Science (CS) about who is considered CS material, and therefore, more likely to succeed or fail in the field; and 2) recommend specific ways that formal CS education can be transformed to better support Black women. Analysis of the 14 interviews provides answers to the following research questions:

(1) How are Black women entering the field of computing? 
(2) What are the intersectional experiences of Black women at the undergraduate and graduate levels in CS education?

(3) What do these experiences suggest about broadening participation in computing?

In this paper, we make three significant contributions to the field of CS education. First, we introduce intersectional computing, a critical framework for analysis in CS education, to explore the lived experiences of Black women, an often overlooked and understudied population in the field of computing. These intersectional experiences of Black women in computing serve as counter-narratives to the dominant narrative about how people come to and move through formal CS education. Finally, our analysis of these lived experiences provides transformative insights to increase the likelihood that Black women will thrive in formal CS education while debunking myths about Black women's ability to succeed in the field of computing.

\section{RELATED WORKS}

Motivated by the need to recruit and retain Black women in computing, this section begins with a brief overview of intersectionality and concludes with an introduction of intersectional computing - a new approach to broadening participation in computing.

\subsection{Overview of Intersectionality}

An intersectional approach becomes necessary when seeking to understand underrepresented or marginalized populations who are often relegated to the outer fringes of society because of the historical and political contexts of racism, gender discrimination, identity politics and White privilege prevalent in the United States [8][9][3][21][20]. Collins \& Bilge[8] define intersectionality as "a way of understanding and analyzing the complexity in the world, in people, and in human experiences...conditions of social and political life and the self can seldom be understood as shaped by one factor. They are generally shaped by many factors in diverse and mutually influencing ways" [p. 2]. In essence, intersectionality takes a multidimensional approach to understanding how social constructs such as race, gender, class, physical ability, sexual orientation, etc. contribute to one's sense of self (Who am I?), one's day to day reality (How do I see the world?) and how others relate to self (How do others see me?).

Before it was first called intersectionality, the origins of intersectionality can be traced to the coalitions of Black women, Chicanos, Native American women, Afro-Brazilian women and other women of color who fought social injustices that denied their humanity and civil rights as far back as the 19th century [7][8][9]. The term intersectionality appears in Crenshaw's [9] work in Critical Race Theory [11], demonstrating the practice of first identifying and then criticizing the power structures that have a negative impact on Black women's well being in society before engaging in acts of resistance against those same power structures. Collins \& Bilge [8] refer to this practice as critical praxis. Intersectionality is not a new concept, having been embraced by the social sciences and humanities as an analytical framework to study indigenous populations in North America, Europe, and the Global South (countries located in Asia, Africa, Latin America and the Caribbean) for hundreds of years. Likewise, because of the acute underrepresentation of women of color (Blacks, Hispanics, LatinX, Native Americans, etc.) in the computing ecosystem, intersectionality has recently surfaced as a critical framework to acquire a deeper understanding of the lived experiences of women of color as they navigate the computing ecosystem, and how these experiences influence their persistence in computing[15][27][40].

\subsection{Intersectional Computing}

Thomas et al.[38] are the first to introduce the concept of intersectional computing - a more complex understanding of the experiences of marginalized groups in computing who live at various intersections of racism, sexism, classism, xenophobia, heterosexism, ableism, etc. Applying intersectionality as a critical praxis advocates for the empowerment of those whose voices have been silenced or ignored. For example, Thomas et al. [38] apply intersectionality as a critical framework to draw attention to the lived experiences of Black women in the field computing. In this example, intersectional computing becomes a tool for promoting diversity, inclusion and equity within the field of computing while shedding light on the plurality of lived intersectional experiences that exist among the broader computing demographic.

The invisibility of Black women "has been critical in maintaining social inequalities"[8][pg. 5]. For example, Black women who programmed mainframes for the NASA Space program only 50 years ago have been erased from the history of computing (along with their contributions) until the nonfictional book Hidden Figures [35] and the 20th Century Fox movie of the same title were released in 2016. This invisibility is indicative of false messages used to explain social inequality within the field of computing. For example, the false message that Black women don't do CS depicts Black women as having little if any interest in CS and serves as justification for their underrepresentation in the field of computing. (Of course, we have heard a similar message about women in general in this Whitemale dominated field [23].) Another false message, White males have a natural predilection for writing code, indicates that women, including women of color, are not naturally imbued with the gift of programming and consequently, CS is not for them[23]. In both of these examples, we can apply intersectional computing to not only understand the micro levels of oppression (i.e. an individual's personal experiences navigating the computing pipeline), but to examine the macro levels of oppression (i.e., White supremacy) that impede the recruitment and retention of marginalized populations in the field of computing.

The exclusion of Black women's ideas, inventions and experiences from mainstream culture which has created an outsider-within standpoint. Such a standpoint positions Black women as being on the periphery of mainstream culture, observing but inherently not a part of a White privileged society[7][16]. For these reasons, we leverage intersectional computing as a mechanism for validating the lived experiences of U.S. Black women in computing while legitimizing their position within the larger computing ecosystem.

\section{METHOD}

\subsection{Position Statement}

Following the methodology of Standpoint Theory [16], we include a statement of standpoint of the research team. The research team 
includes Black women who have strong interests in the disruption of the dominant sociocultural norms in computing. We represent over 18 years of conducting research that aims to promote a more complex narrative of the ways Black women's bodies move through the world, and in computing particularly. In other words, we have a posteriori knowledge about marginalization in computing and STEM spaces. We interchangeably use Black to refer to all women of the African diaspora. Finally, we bracket our various sets of assumptions by including all variations in the Black women's interviews, not rejecting those experiences that may have been divergent from our own.

\subsection{Participants and Settings}

Using the snowball sampling method, we recruited 14 U.S. Black women who were in different stages in the computing pipeline (e.g., graduate students, early career professionals, and junior faculty) to participate in semi-structured interviews.

\subsection{Data Collection and Analysis}

Because of the small sample size $(\mathrm{N}=14)$, we conducted both quantitative and qualitative analyses to better understand this specific population of Black women in computing. The quantitative analysis gauges each participant's current status in the computing pipeline, point of entry, educational experience and background as well as any programming experience prior to matriculation into college. For the qualitative analysis, each semi-structured interview was audio recorded and transcribed for data analysis purposes. Leveraging intersectionality as a critical praxis, we conducted two phases of content analysis of the 14 interviews [36]. In the first phase, all 14 transcripts were imported into NVivo 12. Then, researchers engaged in content analysis in two rounds. First, two researchers independently coded what Black women actually said about their experiences, identifying particular phrases that the interviewees actually uttered that described their experiences as Black women in Computing. As researchers identified actual phrases uttered by the interviewees, they then created a code descriptive of the phrases. The descriptive code (e.g., code - first programming experience) was entered into a code book that contained all of the codes created along with the phrase itself (e.g., phrase - my intro to computer science class was like my first experience programming that corresponded to the code. Next, the two researchers met repeatedly to discuss the rationale for initially identifying 152 unique emergent themes. In the second phase of content analysis, the authors identified similar emergent themes and collapsed them into appropriately named categories (e.g., emergent themes entry-level college CS course, high school CS programming class, CS boot camp, CS summer program, and self-taught) were collapsed into the category of first programming experience, resulting in a set of 29 categories of emergent themes across all 14 interviews.

\section{RESULTS FROM DATA ANALYSIS}

$R Q 1: H o w$ are Black women entering the field of computing? To address this research question, we explore interviewees' current status in the computing pipeline and their educational background, including the different types of computing degrees attained, choice of
Table 1: Computing Degrees

\begin{tabular}{|l||l|}
\hline Type & Number \\
\hline Doctorate & 2 \\
Master's & 9 \\
Bachelor's & 13 \\
Associate & 3 \\
Certificate & 2 \\
\hline
\end{tabular}

institutions for their respective degrees, and their pre-college programming experience. We conclude this section with a preliminary analysis of emergent themes found in the 14 interviews.

\subsection{Current Status in Pipeline}

Participants in our study were successfully navigating the computing pipeline with the heaviest concentration in the industry workforce. Ten of the 14 Black women represented early career professionals employed at private corporations, government agencies, and Fortune 500 companies. Only 1 Black woman represented a tenure-track faculty member at a 4-year institution while 3 Black women were doctoral students currently enrolled at Predominantly White Institutions (PWIs) in the U.S.

\subsection{Computing Degree Attainment}

We assessed the computing degree production among our sample of interviewees. Three of the 14 Black women earned Associate degrees, one in Computer Science, one Engineering Science and the other in Liberal Arts, from three different community colleges. Two of the 14 Black women completed a reputable coding bootcamp and earned a certificate in software development from a PWI. A total of 13 Black women attained their bachelor's degrees with 5 of these participants completing their bachelor's degrees before entering the professional workforce. Seven of the 14 Black women earned their master's degree in computing with 3 of them accepting industry positions. Two Black women had successfully completed their doctoral degrees, with one accepting a faculty position in a CS department and the other doctoral recipient pursuing a technical career in industry.

\subsection{Institution Types for Formal CS Education}

The majority (9 Black women) matriculated into college as incoming CS majors while 6 of them switched their majors to a computing related field after having some exposure to computing (e.g., robotics demonstration, programming course, etc.) within their first year of college. Of our 14 interviewees, less than half of the interviewees attended both Historically Black Colleges or Universities (HBCUs) and Predominantly White Institutions (PWIs) over the course of attaining their undergraduate and graduate degrees. The majority of interviewees graduated with a bachelor's degree in a computing related field from a HBCU compared to only 2 Black women who graduated from a PWI with a similar degree. Note that one Black woman graduated from both a HBCU and a PWI with two bachelor's degrees in computing due to a joint 3-2 engineering program. Additionally, out of the 8 Black women who completed their master's degrees, 6 of them attended PWIs while 2 of the interviewees 
Table 2: Institutions of Formal CS Education

\begin{tabular}{|l||l|l|l|}
\hline $\begin{array}{l}\text { Institution } \\
\text { Type }\end{array}$ & $\begin{array}{l}\text { Bachelor's } \\
\text { Degree }\end{array}$ & $\begin{array}{l}\text { Master's } \\
\text { Degree }\end{array}$ & $\begin{array}{l}\text { Doctoral } \\
\text { Degree }\end{array}$ \\
\hline HBCUs & 9 & 2 & 0 \\
PWIs & 3 & 6 & 4 \\
\hline
\end{tabular}

graduated from HBCUs. Both doctoral recipients attended highly ranked PWIs. Finally, 4 Black women first attended an HBCU for their undergraduate degrees and then a PWI for their graduate degrees. Only 1 participant attended a PWI for her undergraduate degree before completing her master's degree in CS at an HBCU. This is significant because it afforded these interviewees the ability to compare and contrast their experiences across these two different types of institutions.

\subsection{Pre-College Programming Experience}

Most of the Black women (8) admitted to having no prior programming experience before matriculating into college even though 6 of them had some experience with programming in high school courses, summer camps, and as a side hobby that involved creating webpages for family members and friends. An entry level college course was the majority of participants'first introduction to computer programming. This coincides with previous research that indicates that Black girls and women typically suffer from lack of early exposure of CS in grades K-12 despite recent interventions (e.g., CS4All) that target underrepresented populations [31]. Unsurprisingly, most had no clear understanding of the field of computing nor the range of career opportunities available in the field.

\subsection{Thematic Analysis of Black Women's Experiences in CS Education}

RQ2: What are the intersectional experiences of Black women in computing? To answer this question, we identified several emergent themes found in the interviews. Due to page limitations, we shared only a few sample quotes to reflect the most prevalent themes. Note that all identifying information was replaced with pseudonyms.

4.5.1 "I was the only Black female student.". Interviewees often cited instances of being the only Black woman in a traditional classroom setting while pursuing a computing degree at a PWI.

"In general, in my field, and even in undergrad, I was the only black female student in my graduating class, and then when I went to University, I was also - well, there was one other, there was one other black female student, but it was just me and her, and now that I came out here I'm alone again. (laughs)" - Megan

Megan, a doctoral student, had attended only PWIs for her formal CS education, and as such, was used to being the only Black woman in her respective degree programs, with the exception of being one of two in the master's degree program. However, Megan had established a network of support that included Black students on campus to help her feel less isolated. Similar to Garcia \& Scott[15], other interviewees also talked about leveraging their social capital with fellow Black students and Black organizations on campus to create safe havens.

4.5.2 "African American women tend to be left out.". Interviewees also described the lack of Black women included in research studies that sought to increase the representation of women in computing.

"In grad school, then looking into a lot of the women in computing research, I noticed that African American women tend to be left out of those numbers when reading those studies that were done in recent years. Like in the 2000's ...papers that I read in grad school, they didn't think to have a really good representative of African American females when they were doing the studies about women and computing." - Judy

As a graduate student at a PWI, Judy was acutely aware of published research studies that did not include Black women in the sample of participants, and yet these same studies sought to increase the representation of women in the field of computing. Judy's testimony reiterated the previous claim that gender-only focused efforts do little to address the specific issues of being a Black woman in computing[38].

These two emergent themes testified to the social isolation that many interviewees experienced as Black women in computing at PWIs, even though a substantial number of women had matriculated into the graduate programs at these institutions. More importantly, our interviewees spoke about the perception that Black women were often ignored and overlooked, sometimes intentionally, within their respective graduate programs.

4.5.3 "My HBCU prepares you well to handle these situations.". In contrast, interviewees who attended HBCUs described a very different experience as a Black woman in computing.

"So last year I was at Fortune 500 Company...I was the only African American...it didn't like fluster me because I was prepared...My HBCU prepares you well to handle these situations and gives you the confidence to go out and just do the work, keep your head down and be unapologetically a great engineer or whoever you want to be...[HBCU] ingrained in us how to deal with these kinds of problems and have confidence..." Alyssa

Referring to her summer internship experience, Alyssa was not bothered by being the only Black woman working in a particular department at a Fortune 500 company. Instead of feeling isolated or alone, Alyssa was confident that she could do the work and do it well because her educational experiences at a HBCU affirmed her identity as a Black woman and taught her to believe in herself. When sharing her HBCU undergraduate experience, Alyssa talked about having a sense of belonging and where students, though competitive, were also supportive of each other along the way. She, along with other Black women who attended HBCUs, testified about faculty who cared about their students, mentoring and preparing them to confidently enter computing spaces, knowing that they could do the work, solve tough problems and be great computer scientists. (Note: This is not to say that the other two interviewees were less confident because they attended a PWI.) 
4.5.4 "They really, they didn't seem to care as much.". As described previously, 4 of the interviewees experienced formal Computer Science education across a combination of $\mathrm{HBCU}$ and PWI institutions, where interviewees attended attending one type of institution as an undergraduate student and another as a graduate student. Because of their deep engagement with both types of institutions, interviewees described these environments in starkly different ways, especially with regard to how they as Black women in computing were centered, affirmed, and supported.

It was a lot harder for sure. It was more intense. The classroom sizes were bigger. People were different. You know, everybody didn't look like me. And then just the teaching style was different. They didn't really - they didn't seem to care as much as they did at [her HBCU]. They were sort of like 'Come in here, get the information you need, do the homework, do the test.....just being able to connect with the professor was kind of a little bit harder...some professors felt uncomfortable and they don't really know how to interact. And I don't know if that's just like interacting with me or if that was just in general." - Jenny

Jenny was a graduate of a joint 3-2 engineering program between a HBCU and a PWI. She described her experience at her HBCU as being supportive with professors and students who looked like her. She clearly conveyed a sense of family among her peers and instructors as an undergraduate student at a HBCU. In contrast, after she enrolled in the PWI to complete the engineering program, Jenny noticed that some PWI professors seemed less enthused about teaching and more like "paper pushers." Consequently, Jenny adjusted to the differences and devised strategies to successfully complete the 3-2 engineering program.

4.5.5 "If we're all going to fail, we'll all fail together.". This theme confirmed the sense of community and joint ownership in students' success often attributed to HBCUs. When comparing her undergraduate experiences at a HBCU to a PWI, Lana described the differences in how students interacted and supported each other.

"It [HBCU] was very like competitive kind of a feel like, you know, where everybody's like, 'Okay, if we're all going to fail, we'll all fail together'...but there [PWI] it was like, 'Okay, I want my A and I'm going to get my A, but I don't care if you get an A or fail.' So when it came down to like studying between the students it was more...like everybody sort of had their clique I guess you could say. And it wasn't as welcoming as, 'Oh, come study with us.' It was more so trying to... find the right people to study with too who will let you study with them." - Lana

Clearly, Lana encountered a more hostile environment at the PWI, one in which she had to build social capital with students who did not share the same ethnicity or gender as themselves. For Lana and other interviewees who compared their experiences at PWIs, this meant partnering with students who did not look like them but were willing to include them in their study groups or share study materials (e.g., previous exams). In comparison, Lana could rely on her social capital as a student at a HBCU since her fellow classmates shared the same ethnicity, and consequently, the same history of oppression, discrimination and racism that created an immediate sense of community, support and fellowship. It was this same sense of "we're all in this together," that motivated Black women to "keep on pressing on."[38]

4.5.6 "There was a lot of mentoring and guidance in that whole process.". Renee, reflecting upon her undergraduate experience at a HBCU and her graduate experience at a PWI, also described the differences in the willingness of other CS students to work together as well as the ways faculty engaged with students.

"So I would partner up with those students in class who had prior experience. So that's I guess one thing about $[\mathrm{HBCU}]$...it wasn't a competitive environment but more a collaborative environment. So classmates didn't compete with one another but they did work together to better understand and work on assignments and things like that. And there was a lot of mentoring and guidance in that whole process in undergrad...so they were set on mentoring. Open door, you can go to any professor, they read your college applications and that sort of thing. No TAs to go through or anything like that...It [her HBCU] was a really open sort of environment...People weren't afraid to ask questions and say they didn't know something and learn something. So when I first came to [PWI] they automatically started off as a very competitive environment for their students...nobody wants to admit when they don't know something and that sort of thing." - Renee

What became apparent over the course of the interviews was that CS departments at HBCUs fostered a more supportive learning environment in which faculty and students represented a connected social network - one heavily invested in the success of its students. Faculty were easily accessible and spent a considerable amount of time mentoring students in addition to their teaching responsibilities. Interviewees repeatedly reported that the culture of HBCUs was one of collaboration more so than competition. Such an open culture allowed students to admit when they did not understand something without making negative assumptions about their level of intelligence. The stark contrast between the culture of HBCUs versus PWIs was evident, pointing to opportunities to reshape CS education at PWIs.

4.5.7 "I'm not that woke.". Given the contrast between PWIs and HBCUs, how were Black women at PWIs managing to navigate the computing ecosystem? Megan, a doctoral student, shared her strategy for persistence.

"I'm not that woke, and I've kind of trained myself to do that on purpose...I kind of just trained myself to ignore it because...if I always acknowledge it and always recognize it, I would get upset, and in order to prevent myself - like, to protect myself from being upset and to remain a happy and content individual." - Megan

Megan had learned how to cope with racism at a PWI by becoming desensitized to racist remarks from fellow classmates. For example, one interviewee shared how non-Black classmates would engage in 
conversations about recent events which involved police brutality in the African American community, describing them as justifiable actions taken against criminals (i.e., must have done something wrong). She simply chose to ignore such conversations, instead staying focused on getting her doctoral degree. Other interviewees testified to engaging in the same behavior as a coping mechanism.

\section{DISCUSSION}

RQ3:What do these experiences suggest about broadening participation in computing? The authors intentionally place the responsibility of addressing the power structures that disadvantage Black women and other women of color on the very institutions and corporations that have the power to change the status quo in the field of computing, rather than positioning those who have been marginalized as being the source of and the solution to the problem [24]. As such, we offer three key takeaways.

First, additional pedagogical approaches are needed to help students to enter and remain in the field regardless of prior experience. HBCUs approach education from the mindset that the institution is here to help students succeed regardless of background, especially since Blacks have overcome many obstacles, including racism, discrimination, poverty, disenfranchisement, etc. to receive a quality education. If a student has a sincere interest in computing, then easy access to a community of support (faculty and students) and additional resources are readily available to scaffold learning and progression through CS curriculum, even for students who have no prior programming experience [31]. Just as HBCUs have had and continue to have success in preparing, educating, and successfully graduating Black women in computing, PWIs can learn from HBCUs how to do the same at their respective institutions, cultivating a more inclusive and diverse culture in computing.

Second, those who hold and wield power within the field (e.g., White men, Asian men, White women, etc.) must utilize their privilege to act on behalf of Black women and to work with organizations, led by Black women, whose goal is to increase the representation of Black women in computing. Recent statistics indicate a higher percentage of White men attain computing degrees followed by Asian males [40]. Thus, the discipline of computing is still largely White, male, and lacking in diversity. If the goal is to significantly increase the percentage of Black women who persist in computing, then those who hold and wield power within the field must be an active part of the solution. For example, CS departments at PWIs can host Black women (and other women of color) in computing as guest speakers, giving them an opportunity to talk about their work while promoting diversity and challenging perceptions of who does computing. Another example is hiring Black women as tenure track and tenured faculty in computing departments. Likewise, including Black women as members of existing computing professional organizations positions them as stakeholders committed to the success of that particular organization. This is by no means an exhaustive list of possible actions but represents a step in the right direction.

Third, PWIs and corporations need to freely share their resources and establish partnerships with HBCUs and organizations within the Black community. Resources can include information about career opportunities, access to campus facilities, equipment, etc. The critical difference in our solution is that there must be an equal exchange between PWIs and HBCUs. Referring to the participants in our study who talked about their educational experiences at PWIs and work experiences in industry, PWIs and many tech companies continue to perpetuate the White male dominated culture of computing and consequently, do a poor job of recruiting and retaining Black women in computing. Rather than perceiving HBCUs as being subpar to PWIs, PWIs and technology companies need to seek out HBCUs with an attitude of wanting to learn the best practices for increasing the representation of Blacks in computing.

\section{CONCLUSION}

Intersectional computing is not just a matter of recruiting students who can check off multiple boxes (e.g., race, ethnicity, gender, sexuality, etc.) for the purpose of evaluating a CS intervention, pedagogical strategy or learning outcome. Rather, researchers must be held accountable for their use of intersectionality as an analytical tool for studying women of color in computing, refraining from interventions that "add girls, sprinkle programming and stir"[15]. Such an approach represents a misguided use of intersectionality that fails to address the power structures at play within the field of computing[24]. Intentional efforts to create a more inclusive and equitable CS educational environment at PWIs is warranted, especially given the anticipated diversity of future generations of college students. However, more work remains in removing barriers of entry into the field of computing. Intersectional computing can serve as a useful mechanism for transforming the field of computing into one that is "more collaborative than competitive" and indeed open to all.

\section{ACKNOWLEDGMENTS}

This research is generously supported via NSF grant 1937759 and Florida State University grant 138000-551-041995.

\section{REFERENCES}

[1] 2015. Women, Minorities, and Persons with Disabilities in Science and Engineering: 2015 Special Report NSF 15-311. http://www.nsf.gov/statistics/wmpd/

[2] L. Barker. 2009. Student and Faculty Perceptions of Undergraduate Research Experiences in Computing. Trans. Comput. Educ. 9, 1, Article 5 (March 2009), 28 pages. https://doi.org/10.1145/1513593.1513598

[3] bell hooks. 2000. Feminist theory: From margin to center. Pluto Press.

[4] Jamika D. Burge, Jakita O. Thomas, and Ryoko Yamaguchi. 2016. Computing and Intersectionality: The Social and Behavioral Structures at Play for Black Women in the Computing Sciences. Final Workshop Report.

[5] Jamika D. Burge, Jakita O. Thomas, and Ryoko Yamaguchi. 2017. Follow-Up Workshop: Computing and Intersectionality: The social and behavioral structures at play for Black women in the Computing Sciences. Final Workshop Report.

[6] J. McGrath Cohoon and William Aspray. 2006. Retaining women in undergraduate computing. In Women and information technology: Research on underrepresentation. Vol. 1. MIT Press, Cambridge, MA, 137-180.

[7] Patricia Hill Collins. 2000. Black Feminist Thought: knowledge, consciousness, and the politics of empowerment. Routledge Taylor \& Francis Group, New York and London.

[8] Patricia Hill Collins and Sirma Bilge. 2016. Intersectionality. Polity Press.

[9] Kimberle Crenshaw. 1989. Demarginalizing the Intersection of Race and Sex: A Black Feminist Critique of Antidiscrimination Doctrine, Feminist Theory and Antiracist Policies. 1 (1989), 139-167.

[10] Kimberle Crenshaw. 1993. Demarginalizing the Intersection of Race and Sex: A Black Feminist Critique of Antidiscrimination Doctrine, Feminist Theory and Antiracist Politics.

[11] Kimberle Crenshaw, Neil Gotanda, Gary Peller, and Kendall Thomas. 1995. Critical Race Theory: The key writings that formed the movement. The New Press.

[12] Ron Eglash, William Babbitt, Audrey Bennett, Kathryn Bennett, Brian Callahan, James Davis, John Drazan, Charles Hathaway, David Hughes, Mukkai Krishnamoorthy, Michael Lachney, Michael Mascarenhas, Shayla Sawyer, and Kathleen 
Tully. 2017. Culturally Situated Design Tools: Generative fustice as a Foundation for STEM Diversity. IGI Global Publishers, Hershey, PA.

[13] Ron Eglash, Juan E Gilbert, and Ellen Foster. 2013. Toward culturally responsive computing education. Commun. ACM 56, 7 (2013), 33-36.

[14] Carol Frieze, Orit Hazzan, Lenore Blum, and M. Bernardine Dias. 2006. Culture and Environment As Determinants of Women's Participation in Computing: Revealing the "women-CS Fit". In Proceedings of the 37th SIGCSE Technical Symposium on Computer Science Education (SIGCSE '06). ACM, New York, NY, USA, 22-26. https://doi.org/10.1145/1121341.1121351

[15] Patrica Garcia and Kimberly Scott. 2016. Traversing a Political Pipeline: An Intersectional and Social Constructionist Approach Toward Technology Education for Girls of Color. InterActions: UCLA fournal of Education and Information Studies 12, 2 (2016), 1-26.

[16] Sandra G. Harding. 1992. Rethinking standpoint epistemology: What is strong objectivity? Centennial Review 36, 3 (1992), 437-470.

[17] Catherine Hill, Christianne Corbett, and Andresse St. Rose. 2010. Why So Few? Women in Science, Technology, Engineering, and Mathematics.

[18] Apriel Hodari, Maria Ong, Lily T. Ko, and R. R. Kachchaf. 2014. New Enactments of Mentoring and Activism U.S. Women of Color in Computing Education and Careers. In In Proceedings of the Tenth annual conference on International Computing Education Research. 83-90.

[19] Apriel K. Hodari, Maria Ong Lily T. Ko, and Janet Smith. 2015. 2015 Research in Equity and Sustained Participation in Engineering, Computing, and Technology (RESPECT) Enabling courage: Agentic strategies of women of color in computing. 1 (2015), 1-7. 10.1109/RESPECT.2015.7296497

[20] Yannick St. Jean and Joe R. Feagin. 1998. Double Burden: Black women and everyday racism. M.E. Sharpe.

[21] Lindsey Malcom and Shirley Malcom. 2011. The double bind: The next generation Harvard Educational Review 81, 2 (2011), 162-172.

[22] Jane Margolis. 2010. Stuck in the Shallow End: education, race, and computing. MIT Press.

[23] Jane Margolis and Allan Fisher. 2003. Unlocking the clubhouse: Women in computing. MIT Press.

[24] Terrell R. Morton and Eileen C. Parsons. 2018. BlackGirlMagic: The identity conceptualization of Black women in undergraduate STEM education. Science Education 102 (2018), 1363-1393.

[25] Maria Ong. 2011. The Status of Women of Color in Computer Science. Commun. ACM 54, 7 (July 2011), 1-3.

[26] Maria Ong, Janet M. Smith, and Lily T. Ko. 2017. Counterspaces for women of color in STEM higher education: Marginal and central spaces for persistence and success. Journal of Research in Science Teaching 5, 2 (2017), 206-245. https: //doi.org/10.1002/tea.21417

[27] Maria Ong, Carol Wright, Lorelle L. Espinosa, and Gary Orfield. 2011. Inside the double bind: A synthesis of empirical research on undergraduate and graduate women of color in science, technology, engineering, and mathematics. Harvard Educational Review 81, 2 (2011), 172-208.
[28] Nichole Pinkard, Sheena Erete, Caitlin Kennedy Martin, and Maxine McKinney de Royston. 2017. Digital Youth Divas: Exploring narrative-driven curriculum to spark middle school girls' interest in computational activities. Fournal of the Learning Sciences 26, 3 (2017), 477-516.

[29] Nichole Pinkard, C. K. Martin, and S. Erete. 2019. Equitable approaches: opportunities for computational thinking with emphasis on creative production and connections to community. Interactive Learning Environments 0, 0 (2019), 1-15. https://doi.org/10.1080/10494820.2019.1636070 arXiv:https://doi.org/10.1080/10494820.2019.1636070

[30] Yolanda A. Rankin and Jakita O. Thomas. 2017. Leveraging Food as the Context for Developing Computational Algorithmic Thinking in an Entry Level College Course. IGI Global Publishers, Hershey, PA.

[31] Yolanda A. Rankin, Jakita O. Thomas, and India Irish. 2019. Food for Thought: Supporting African American Women's Computational Algorithmic Thinking in an Intro CS Course. In In Proceedings of the Special Interest Group ACM Symposium on Computer Science Education (SIGCSE '19). ACM Press, 641-646.

[32] Penny Rheingans, Crystal Dias-Espinoza, Erica D’Eramo, and Danyelle Ireland. 2018. A Model for Increasing Gender Diversity in Technology. In In Proceedings of ACM Special Interest Group on Computer Science Education Conference (SIGCSE 18). ACM Press, Baltimore, MD USA, 459-464.

[33] Kimberly Scott, Kimberly Sheridan, and Kenneth Clark. 2014. Culturally Responsive Computing: a theory revisited. Learning Media and Technology 40, 4 (December 2014), 1-25. 10.1080/17439884.2014.924966

[34] Kimberly Scott and Mary Aleta White. 2013. CompuGirls' Standpoint: Culturally Responsive Computing and Its Effect on Girls of Color. Urban Education 48, 5 (September 2013), 657-681.

[35] Margot Lee Shetterly. 2016. Hidden Figures: The American Dream and the Untold Story of the Black Women Mathematicians Who Helped Win the Space Race. HarperCollins, New York, NY.

[36] Anselm L. Strauss and Juliet Corbin. 1990. Basics of qualitative research: Grounded theory procedures and techniques. Sage Publications, Newbury Park, California.

[37] Jakita O. Thomas. 2018. The Computational Algorithmic Thinking (CAT) Capability Flow: an approach to articulating CAT Capabilities over Time in AfricanAmerican Middle-School Girls. In In Proceedings of ACM Special Interest Group on Computer Science Education Conference (SIGCSE '18). ACM Press, Baltimore, MD USA, 149-154

[38] Jakita O. Thomas, Nicole Joseph, Arian Williams, Chan'tel Crum, and Jamika Burge. 2018. Speaking Truth To Power: Exploring the Intersectional Experiences of Black women in Computing. In Proceedings of the 3rd Annual Conference for Research On Equity, Sustained Participation in Computing, Engineering \& Technology (RESPECT '18), J. Payton and G. Thiruvathukal (Eds.). Baltimore, MD, USA.

[39] Ryoko Yamaguchi and Jamika D. Burge. 2019. Intersectionality in the narratives of black women in computing through the education and workforce pipeline. fournal for Multicultural Education 13, 3 (2019), 215-235.

[40] Stuart Zweben and Betsy Bizot. 2019. 2018 CRA Taulbee Survey. 31, 5 (2019), $3-74$. 